\title{
ON THE DISTORTION IN CONFORMAL MAPPING OF VARIABLE DOMAINS( $\left.{ }^{(}\right)$
}

\author{
BY \\ S. E. WARSCHAWSKI \\ INTRODUCTION
}

For many problems in conformal mapping it is of importance to have precise quantitative information concerning the variation of the mapping function of a region onto a circle, under a deformation of the region. A number of estimates for this variation in terms of parameters, which measure the deformation and the smoothness of the boundary of the region, have been given in the literature. A survey of such results is contained in [13] and $[11]\left({ }^{2}\right)$.

While the degree of the change in the mapping function itself has been investigated for various general configurations, the corresponding question concerning the distortion, i.e. the derivative of the mapping function, has been studied primarily for nearly circular regions. The object of the present paper is to obtain such estimates of the distortion for arbitrary regions under quite general hypotheses.

The paper is divided into two parts. The question considered in the first part arose in connection with the following theorem [8, p. 364]. Suppose $C$ and $C_{n}(n=1,2, \cdots)$ denote closed Jordan curves which are represented by the equations

$$
w=w(t) \text { and } \quad w=w_{n}(t), \quad 0 \leqq t \leqq 1,
$$

respectively, where $w(t), w_{n}(t)$ are periodic with the period 1 , have continuous nonvanishing derivatives, and

$$
w_{n}(t) \rightarrow w(t), \quad w_{n}^{\prime}(t) \rightarrow w^{\prime}(t), \quad \text { as } n \rightarrow \infty,
$$

uniformly for $0 \leqq t \leqq 1$. If $f(z)$ and $f_{n}(z)$ map the circle $|z|<1$ conformally. onto the interiors of $C$ and $C_{n}$, respectively, such that $f(0)=f_{n}(0)$ and $f^{\prime}(0)$ $>0, f_{n}^{\prime}(0)>0$, then, for every $p>0$,

$$
\lim _{n \rightarrow \infty} \mathfrak{M i}_{p}\left\{f^{\prime}-f_{n}^{\prime}\right\}=\lim _{n \rightarrow \infty}\left[\frac{1}{2 \pi} \int_{0}^{2 \pi}\left|f_{n}^{\prime}\left(e^{i \theta}\right)-f^{\prime}\left(e^{i \theta}\right)\right|^{p} d \theta\right]^{1 / n}=0 .
$$

We supplement this result here in a quantitative sense by giving an estimate for the rate of convergence of this integral. Let $C$ and $C_{1}$ be two closed Jordan

Presented to the Society, September 2, 1955; received by the editors November 14, 1955.

(1) This work was performed under contract $\mathrm{N}$ onr-710(16) (NR 044 004) between the University of Minnesota and the Office of Naval Research.

(2) Numbers in the brackets indicate the literature references at the end of this paper. 
curves with continuously turning tangent and let $f(z)$ and $f_{1}(z)$ be the corresponding mapping functions defined and normalized as $f$ and $f_{n}$ above. Then we obtain a bound for $\mathfrak{M}_{p}\left\{f^{\prime}-f_{1}^{\prime}\right\}$ in terms of the "deviation" of $C_{1}$ from $C$ and of other parameters which characterize certain geometric properties of $C$ and $C_{1}$ (Theorem I). This result generalizes a previous theorem [14] which was stated and proved only for a special form of the modulus of continuity of the tangent angles of $C$ and $C_{1}$. The present result is general.

The second part of the paper deals with estimates of the actual difference of the derivatives, i.e. the $\operatorname{Max}_{|z| \leqq 1}\left|f^{\prime}(z)-f_{1}^{\prime}(z)\right|$, and of a similar expression of the derivatives of the inverse functions. These bounds are of the same type as those for the $L^{p}$ mean, and the fact that they depend only on certain parameters characterizing the geometrical configuration, and in no other way on $C$ and $C_{1}$, makes the results applicable not only to a given pair but also to a family of pairs $C, C_{1}$, which pertain to the same parameter values.

\section{Estimates for the $L^{p}$ Mean}

1. Statement of results. Suppose $C$ and $C_{1}$ denote two closed Jordan curves in the $w$-plane which possess continuously turning tangents. Let $\tau(s)$, $\tau_{1}(s)$ denote their tangent angles, expressed as functions of the arc length, and let $\beta(t)$ and $\beta_{1}(t)$ be moduli of continuity for $\tau(s)$ and $\tau_{1}(s)$, respectively, i.e. $\beta(t)$ is a monotone function, defined for $t>0$, with $\lim _{t \rightarrow 0} \beta(t)=0$, such that

$$
|\tau(s \pm h)-\tau(s)| \leqq \beta(h), \quad 0 \leqq s \leqq L, 0<h \leqq L,
$$

where $L$ is the total length of $C$. Here $\tau(s)$ may be chosen arbitrarily mod $2 \pi$ and $\tau(s \pm h)$ is so determined that $\tau(s \pm x)$ is continuous for $0 \leqq x \leqq h$. Throughout the paper $C$ and $C_{1}$ will be subject to some or all of the following assumptions:

(i) $C$ and $C_{1}$ are contained in the ring $0<d \leqq|w| \leqq D$.

(ii) If $\Delta s$ denotes the (shorter) arc of either curve between $w^{\prime}$ and $w^{\prime \prime}$, then

$$
\frac{\Delta s}{\left|w^{\prime}-w^{\prime \prime}\right|} \leqq c .
$$

(iii) For some $\epsilon>0, C_{1}$ is in the $\epsilon$-neighborhood of $C$, i.e. every point of $C_{1}$ is contained in a circle of radius $\epsilon$ about some point of $C$.

(iv) $C$ is in the $\epsilon$-neighborhood of $C_{1}\left({ }^{3}\right)$.

(v) To every point $w_{1} \in C_{1}$ associated with the value $s_{1}$ of the arc length, $0 \leqq s_{1} \leqq L_{1}$, there corresponds a point $w \in C$, pertaining to the arc length value $\sigma=\sigma\left(s_{1}\right)$, such that $\left|w_{1}-w\right| \leqq \epsilon$ and that, for suitable choice of the branches,

(3) Condition (iii) does not imply (iv). However, it can be shown that (iii) together with (i) and (ii) imply that $C$ lies within an $\epsilon_{1}$-neighborhood of $C_{1}$ where $\epsilon_{1} \leqq k \epsilon$ and $k$ is a constant which depends only on $d, D$, and $c$. The writer owes a proof for this fact to Fulton Koehler [4]. Inasmuch as it would be sufficient for our purposes to know that $C$ is contained in an $\epsilon_{1}$ neighborhood of $C_{1}$ with $\epsilon_{1}=k \epsilon$, the condition (iv) is not essential. 


$$
\eta=\operatorname{Sup}_{0 \leqq s_{1} \leqq L_{1}}\left|\tau_{1}\left(s_{1}\right)-\tau\left(\sigma\left(s_{1}\right)\right)\right| \leqq \frac{\pi}{2}-\alpha<\pi / 2 .
$$

REMARKs. 1. If $C$ and $C_{1}$ satisfy hypotheses (i)-(v) and if $\epsilon$ is so small that $\beta(2 \epsilon c) \leqq \alpha / 2$, then condition (v), holds also if the roles of $C$ and $C_{1}$ are interchanged and $\alpha$ replaced by $\alpha / 2$.

For any $w \in C$ there exists by (iv) a point $w_{1} \in C_{1}$ such that $\left|w-w_{1}\right| \leqq \epsilon$. We denote by $\tau^{*}(w)$ (and $\tau_{1}^{*}\left(w_{1}\right)$ ) the tangent angle to $C$ at $w\left(C_{1}\right.$ at $\left.w_{1}\right)$. By (v) there corresponds to $w_{1} \in C_{1}$ a point $w^{\prime} \in C$ such that $\left|w_{1}-w^{\prime}\right| \leqq \epsilon$ and $\left|\tau_{1}^{*}\left(w_{1}\right)-\tau^{*}\left(w^{\prime}\right)\right| \leqq \pi / 2-\alpha$. Now the length of the shorter arc of $C$ between $w$ and $w^{\prime}$ is at most $c \cdot\left|w-w^{\prime}\right| \leqq 2 c \epsilon$. Hence, for suitable choice of the angle $\tau^{*}(w)(\bmod 2 \pi),\left|\tau^{*}(w)-\tau^{*}\left(w^{\prime}\right)\right| \leqq \beta(2 c \epsilon) \leqq \alpha / 2$ and thus

$$
\left|\tau^{*}(w)-\tau_{1}^{*}\left(w_{1}\right)\right| \leqq \frac{\pi}{2}-\frac{\alpha}{2} .
$$

On the other hand, if the branch of $\tau^{*}(w)$ is prescribed, that of $\tau^{*}\left(w^{\prime}\right)$ and $\tau_{1}^{*}\left(w_{1}\right)$ may be simultaneously so adjusted $(\bmod 2 \pi)$ that $(1.1)$ holds. This proves our statement.

2. Conditions (iii), (iv) and (v) are clearly satisfied if $C$ and $C_{1}$ are represented in parametric form by the equations

$$
w=w(t) \text { and } w_{1}=w_{1}(t), \quad 0 \leqq t \leqq a,
$$

respectively, such that, $\left|w^{\prime}(t)\right| \geqq b>0,\left|w_{1}^{\prime}(t)\right| \geqq b$ and

$$
\left|w(t)-w_{1}(t)\right| \leqq \epsilon, \quad\left|w^{\prime}(t)-w_{1}^{\prime}(t)\right| \leqq \delta,
$$

if $\delta$ is sufficiently small (one can show that $\eta \leqq \pi \delta / 2 b$ ).

Suppose $w=f(z)$ and $w=f_{1}(z)$ map $|z|<1$ conformally onto the interiors of $C$ and $C_{1}$, respectively, such that $f(0)=f_{1}(0)=0$ and $f^{\prime}(0)>0, f_{1}^{\prime}(0)>0$.

Our principal result here is the following theorem.

THEOREM I. Under the assumptions (i)-(v) there exist, for every $p>1$ :

(1) a constant $M_{p}$, such that $\left({ }^{4}\right)$

$$
\mathfrak{M}_{p}\left\{f-f_{1}\right\}=\left[\frac{1}{2 \pi} \int_{0}^{2 \pi}\left|f\left(e^{i \theta}\right)-f_{1}\left(e^{i \theta}\right)\right| p d \theta\right]^{1 / p} \leqq M_{p} \epsilon ;
$$

(2) a constant $K_{p}$ such that

$$
\mathfrak{M}_{p}\left\{f^{\prime}-f_{1}^{\prime}\right\} \leqq K_{p}\left[\eta+\beta^{*}\left(2 c M_{p} \epsilon\right)\right]
$$

where $\beta^{*}(t)$ is any convex majorant of $\beta(t)$ in a neighborhood of $t=0$, i.e. $\beta^{*}(t)$ $\geqq \beta(t), \beta^{*}(t)$ is convex from above and $\lim _{t \rightarrow 0} \beta^{*}(t)=0$. The constants $M_{p}$ and $K_{p}$ depend only on $p, d, D, c, \alpha$ and the functions $\beta_{i}(t)$.

(4) An estimate in terms of a lower order in $\epsilon, \mathfrak{D}_{p}\left\{f-f_{1}\right\}=O\left(\epsilon^{1 / 8}\right)$, was obtained by F. J. Polansky (see $\left[13\right.$, p. 184]) under weaker assumptions on $C$ and $C_{1}$. 
Part (2) contains as a special case a result which was established previously [14]:

CoRollaRY. If $\beta(t)=H t^{\gamma}, 0<\gamma \leqq 1$, and $\eta=q \epsilon^{\gamma}$, where $H$ and $q$ are constants, then

$$
\mathfrak{M}_{p}\left\{f_{1}^{\prime}-f^{\prime}\right\} \leqq N_{p} \epsilon^{\gamma},
$$

where $N_{p}$ depends only on $p, d, D, c, \gamma, H$, and $q$.

2. Lemmas. For the proof of this theorem we shall need three lemmas. Before stating these we introduce the following convention to be adopted throughout the paper. Suppose, as above, that $C$ is a closed Jordan curve with continuously turning tangent, $\beta(t)$ a modulus of continuity of the tangent angle $\tau(s), w=f(z)$ the mapping function described. Let, for any real $\theta$, $s(\theta)=\int_{0}^{\theta}\left|f^{\prime}\left(e^{i t}\right)\right| d t$. Then we shall mean by $\tau[s(\theta)]$ the branch of this angle for which

$$
\tau[s(\theta)]=\arg f^{\prime}\left(e^{i \theta}\right)+. \theta+\frac{\pi}{2},
$$

where $\arg f^{\prime}(0)=0$. Since $\arg f^{\prime}(z)$ is single-valued and continuous in $|z| \leqq 1$, $\tau[s(\theta)]-\theta$ is a single-valued and continuous function and, for any $\theta_{1}, \theta_{2}$,

$$
\left|\tau\left[s\left(\theta_{1}\right)\right]-\tau\left[s\left(\theta_{2}\right)\right]\right| \leqq \beta\left(\left|s\left(\theta_{1}\right)-s\left(\theta_{2}\right)\right|\right),\left|\theta_{2}-\theta_{1}\right| \leqq 2 \pi .
$$

We turn now to the three lemmas.

Lemma 1. Suppose $C$ is the curve described in $\$ 1$, which satisfies hypotheses (i) and (ii), and $w=f(z)$ is the function defined above. Then there exist two constants $m_{1}$ and $m_{2}$ which depend only on $d, D, c$ and the function $\beta(t)$ such that for $\left|z_{1}\right|=1,\left|z_{2}\right| \leqq 1$

$$
m_{1}\left|z_{1}-z_{2}\right|^{2} \leqq\left|f\left(z_{1}\right)-f\left(z_{2}\right)\right| \leqq m_{2}\left|z_{1}-z_{2}\right|^{1 / 2},
$$

and if $s(\theta)=\int_{0}^{\theta}\left|f^{\prime}\left(e^{i t}\right)\right| d t$,

$$
\left|s\left(\theta_{2}\right)-s\left(\theta_{1}\right)\right| \leqq m_{2}\left|\theta_{2}-\theta_{1}\right|^{1 / 2} .
$$

Furthermore, there exists a function $\delta(x), 0<x \leqq 1$, with $\lim _{x \rightarrow 0} \delta(x)=0$ which depends only on $\beta\left(m_{2} t^{1 / 2}\right)$ such that, for $0 \leqq \theta \leqq 2 \pi$,

$$
\left|\arg f^{\prime}\left(r e^{i \theta}\right)-\arg f^{\prime}\left(e^{i \theta}\right)\right| \leqq \delta(1-r) \quad\left(\arg f^{\prime}(0)=0\right) .
$$

Finally, if $w$ is in the interior $I(C)$ of $C$ and $|w| \geqq d$, then the inverse function of $f(z)$ maps $w$ onto a point $z$ such that $|z| \geqq d / D$.

Proof. The inequalities (2.3) and (2.4) follow immediately from [12, p. 255]. The function $\delta(x)$ is easily determined and (2.5) established by use of the Poisson integral representation of $\arg f^{\prime}(z)$ in $|z|<1$. One merely needs to observe that $\beta\left(m_{2} t^{1 / 2}\right)+t$ is a modulus of continuity for $\arg f^{\prime}\left(e^{i \theta}\right)$ and that 
$\mathfrak{M}_{1}\left\{\arg f^{\prime}\left(e^{i \theta}\right)\right\}=\arg f^{\prime}(0)=0$. The last statement of the lemma follows by application of Lindelöf's principle to the functions $w / D$ and $f^{-1}(w)$.

Lemma 2. Suppose $C$ and $w=f(z)$ have the same meaning as in Lemma 1. Let $w_{0}=f\left(e^{i \theta}\right), w_{1}=f\left(r e^{i \theta}\right)$, for some $r, 0<r<1$, and let $\omega$ be the shortest distance of $w_{1}$ from $C$. Suppose $\left|w_{1}\right| \geqq d$. Let $\lambda$ denote the length of the arc $l: w=f\left(\rho e^{i \theta}\right)$, $r \leqq \rho \leqq 1$. Then there exists an $\epsilon_{0}>0$ which depends only on $d, D, c$ and the function $\beta(t)$ such that if $\omega \leqq \epsilon_{0}$,

$$
\lambda \leqq 2 \omega .
$$

Proof. The tangent angle at any point $f\left(\rho e^{\theta \theta}\right)$ of $l$ is given by

$$
\mu(\rho, \theta)=\theta+\arg f^{\prime}\left(\rho e^{i \theta}\right), \quad 0 \leqq \theta \leqq 2 \pi .
$$

Let $w_{2}$ be a point on $C$ closest to $w_{1}$ so that $\omega=\left|w_{2}-w_{1}\right|$. The direction angle of the segment $w_{1} w_{2}$, which is normal to $C$ at $w_{2}$, is

$$
\mu=\theta_{2}+\arg f^{\prime}\left(e^{i \theta_{2}}\right),
$$

where $\theta_{2}$ is determined by $w_{2}=f\left(e^{i \theta_{2}}\right)$ and is so chosen that $\left|\theta-\theta_{2}\right| \leqq \pi$. Hence

$$
\begin{aligned}
\mu(\rho, \theta)-\mu & =\theta-\theta_{2}+\arg f^{\prime}\left(\rho e^{i \theta}\right)-\arg f^{\prime}\left(e^{i \theta}\right)+\arg f^{\prime}\left(e^{i \theta}\right)-\arg f^{\prime}\left(e^{i \theta_{2}}\right) \\
& =\tau[s(\theta)]-\tau\left[s\left(\theta_{2}\right)\right]+\arg f^{\prime}\left(\rho e^{i \theta}\right)-\arg f^{\prime}\left(e^{i \theta}\right) .
\end{aligned}
$$

Hence, by (2.2) and by Lemma 1, (2.4) and (2.5):

$$
\begin{aligned}
|\mu(\rho, \theta)-\mu| & \leqq \beta\left(\left|s(\theta)-s\left(\theta_{2}\right)\right|\right)+\delta(1-r) \\
& \leqq \beta\left(m_{2}\left|\theta-\theta_{2}\right|^{1 / 2}\right)+\delta(1-r) .
\end{aligned}
$$

We obtain from (2.3)

$$
(1-r)^{2} \leqq\left|r e^{i \theta}-e^{i \theta_{2}}\right|^{2} \leqq \omega / m_{1},
$$

and since $r\left|e^{i \theta}-e^{i \theta_{2}}\right| \leqq\left|r e^{i \theta}-e^{i \theta_{2}}\right|$ we have, because of the assumption $\left|w_{1}\right| \geqq d$ and the last part of Lemma 1 ,

$$
\left|\theta-\theta_{2}\right| \leqq \frac{\pi}{2}\left|e^{i \theta}-e^{i \theta_{2}}\right| \leqq \frac{\pi}{2 r}\left(\frac{\omega}{m_{1}}\right)^{1 / 2} \leqq \frac{\pi}{2} \frac{D}{d}\left(\frac{\omega}{m_{1}}\right)^{1 / 2}
$$

Thus, from (2.6),

$$
|\mu(\rho, \theta)-\mu| \leqq \beta\left(\frac{m_{2} \pi D}{2 d}\left(\frac{\omega}{m_{1}}\right)^{1 / 4}\right)+\delta\left(\left(\frac{\omega}{m_{1}}\right)^{1 / 2}\right) .
$$

Hence we can determine an $\epsilon_{1}>0$ which depends only on $d, D, c$, and $\beta(t)$ such that the right-hand side of this inequality does not exceed $\pi / 8$ if $\omega \leqq \epsilon_{1}$. This implies that the tangent to any point of $l$ forms an angle $\leqq \pi / 8$ with the normal $w_{1} w_{2}$ to $C$. Hence $l$ lies within an angle $A_{w_{1}}$ of opening $\pi / 4$ with vertex at $w_{1}$, which is bisected by the line $w_{1} w_{2}$. 
There exists an $\epsilon_{2}>0$, which depends only on $d, c$ and $\beta(t)$ such that the circle of radius $\epsilon_{2}$ about any point $w$ of $C$ contains only such points of $C$ in its interior which lie in the angle $B_{w}$ of opening $\pi / 4$ with vertex at $w$ and symmetrical about the tangent to $C$ at $w\left({ }^{5}\right)$. By (2.3) and (2.7), $\left|w_{2}-w_{0}\right|$ $\leqq m_{2}(D / d)^{1 / 2}\left(\omega / m_{1}\right)^{1 / 4}=k \omega^{1 / 4}$. We take now $\epsilon_{0}=\min \left(\epsilon_{1},\left[\epsilon_{2} / k\right]^{4}\right), \omega=\left|w_{1}-w_{2}\right|$ $\leqq \epsilon_{0}$. The point $w_{0}$ at which $l$ and $C$ intersect must lie within the angles $A_{w_{1}}$ and $B_{w_{2}}$, and a simple trigonometric calculation shows that

$$
\left|w_{0}-w_{2}\right| \leqq \frac{\omega}{2} \sec \frac{\pi}{8} .
$$

Furthermore, the projection of $l$ onto the line $w_{1} w_{2}$ does not exceed

$$
\omega+\frac{\omega}{2} \sec \frac{\pi}{8} \cdot \sin \frac{\pi}{8}=\omega\left(1+\frac{1}{2} \tan \frac{\pi}{8}\right) .
$$

Finally, since the tangent line at any point of $l$ forms an angle with the line $w_{1} w_{2}$ which is $\leqq \pi / 8$, we have

$$
\lambda \leqq \omega\left(1+\frac{1}{2} \tan \frac{\pi}{8}\right) \sec \frac{\pi}{8}<2 \omega .
$$

Lemma 3. Suppose $C$ and $C_{1}$ satisfy hypotheses (i), (ii), (iii), and (v) of $\$ 1$. Let $G$ denote the largest subregion common to the interiors of both curves, which contains $w=0$. Then there exists an $\epsilon_{1}$ which depends only on $d, D, c, \alpha$ and the function $\beta(t)$ such that, if $\epsilon \leqq \epsilon_{1}$ :

(a) the image $\Gamma$ of $G$ by means of the inverse function of $w=f(z)$ is starshaped with respect to $z=0$;

(b) if $z=h(\zeta)$ maps $|\zeta|<1$ conformally onto $\Gamma$ such that $h(0)=0, h^{\prime}(0)>0$, then for every $q<\pi(\pi-\alpha)^{-1}$

$$
\mathfrak{M}_{q}\left\{\left|\frac{\zeta h^{\prime}(\zeta)}{h(\zeta)}\right|^{ \pm 1}\right\} \leqq\left[\frac{2}{\cos q(\pi / 2-\alpha / 2)}\right]^{1 / q}, \quad \zeta=\rho e^{i \phi}, 0<\rho \leqq 1 ;
$$

(c) for every $p>1$ there exists a constant $H_{p}$ which depends only on $p, d, D, c$, $a$, and $\beta(t)$, such that

$$
\mathfrak{M}_{p}\{h(\zeta)-\zeta\} \leqq H_{p} \epsilon .
$$

Proof. (a) By [7, p. 87] the boundary of $G$ is a closed Jordan curve and hence the boundary of $\Gamma$ is a closed Jordan curve in $|z| \leqq 1$. This curve is composed of $\operatorname{arcs} \gamma_{k}$ which are images of arcs of $C_{1}$ and therefore lie in $|z|<1$ (except for their endpoints) and of point sets which are images of parts of $C$ and hence are on $|z|=1$.

(5) See, for example [12, p. 256]; the first part of the proof of Lemma 1 in that paper proves our statement concerning $\epsilon$. 
We denote as in $\S 1$ by $\tau_{1}^{*}(w)\left(\tau^{*}(w)\right)$ the tangent angle to $C_{1}$ (or $C$ ) at the point $w$. Suppose now that $z=r e^{i \theta}$ is an (interior) point of an arc $\gamma_{k}$. Since $\gamma_{k}$ possesses a continuously turning tangent, the angle between the normal to $\gamma_{k}$ and the radius at $z$ is given by

$$
v(z)=\tau_{1}^{*}\left[f\left(r e^{i \theta}\right)\right]-\arg f^{\prime}\left(r e^{i \theta}\right)-\frac{\pi}{2}-\theta,
$$

where $\tau_{1}^{*}\left[f\left(r e^{i \theta}\right)\right]$ is, of course, determined only $\bmod 2 \pi$. By hypothesis there exists a point $w^{\prime}=f\left(e^{i \theta^{\prime}}\right)$ on $C$ such that, for $w_{1}=f\left(r e^{i \theta}\right),\left|w_{1}-w^{\prime}\right| \leqq \epsilon$ and

$$
\left|\tau_{1}^{*}\left(w_{1}\right)-\tau^{*}\left(w^{\prime}\right)\right| \leqq \eta \leqq \frac{\pi}{2}-\alpha .
$$

We may assume $\left|\theta^{\prime}-\theta\right| \leqq \pi$. We choose the branch of $\tau^{*}\left(w^{\prime}\right)=\tau\left[s\left(\theta^{\prime}\right)\right]$ in accordance with (2.1), and (2.10) determines then the choice of $\tau_{1}^{*}\left(w_{1}\right)$. We obtain thus

$$
v(z)=\tau_{1}^{*}\left[f\left(r e^{i \theta}\right)\right]-\tau\left[s\left(\theta^{\prime}\right)\right]+\tau\left[s\left(\theta^{\prime}\right)\right]-\tau[s(\theta)]+\arg f^{\prime}\left(e^{i \theta}\right)-\arg f^{\prime}\left(r e^{i \theta}\right) .
$$

Hence, by (2.2) and (2.5),

$$
|v(z)| \leqq \eta+\beta\left(\left|s\left(\theta^{\prime}\right)-s(\theta)\right|\right)+\delta(1-r) .
$$

If $\epsilon \leqq \epsilon_{0}$, the bound of Lemma 2, then, since $w_{1}$ has at most the distance $\epsilon$ from $C$, we have by this lemma, $\left|f\left(r e^{i \theta}\right)-f\left(e^{i \theta}\right)\right| \leqq \lambda \leqq 2 \epsilon$. Hence, by (2.3),

$$
(1-r)^{2} \leqq \frac{1}{m_{1}}\left|f\left(r e^{i \theta}\right)-f\left(e^{i \theta}\right)\right| \leqq \frac{2 \epsilon}{m_{1}} .
$$

Furthermore (see (2.7))

$$
\left|\theta^{\prime}-\theta\right| \leqq \frac{\pi}{2}\left[\frac{\left|f\left(e^{i \theta^{\prime}}\right)-f\left(e^{i \theta}\right)\right|}{m_{1}}\right]^{1 / 2} \leqq \frac{\pi}{2}\left(\frac{3 \epsilon}{m_{1}}\right)^{1 / 2} .
$$

We can determine, therefore, an $\epsilon_{1} \leqq \epsilon_{0}$, which depends only on $d, D, c, \alpha$, and $\beta(t)$ such that, if $\epsilon \leqq \epsilon_{1}$,

$$
|v(z)| \leqq \frac{\pi}{2}-\frac{\alpha}{2}
$$

This inequality implies in particular that any radius drawn to a point of $\gamma_{k}$ can intersect $\gamma_{k}$ in only one point. Any radius ending at a boundary point of $\Gamma$ which is on $|z|=1$ has again only one point in common with the boundary. Thus $\Gamma$ is star-shaped with respect to $z=0$.

(b) Let $V(\zeta)=\arg \left[\zeta h^{\prime}(\zeta) / h(\zeta)\right]$ for $0<|\zeta|<1, V(0)=0$. Then

$$
|V(\zeta)| \leqq \pi / 2 \text { for }|\zeta|<1 \text {. }
$$


$V(\zeta)$ has, therefore, radial boundary values $\psi(\phi)$ almost everywhere on $|\zeta|=1$. On arcs of $|\zeta|=1$ which correspond to the (open) $\gamma_{k}$ by means of the transformation $z=h(\zeta), \psi(\phi)$ is continuous and, by (2.12),

$$
|\psi(\phi)| \leqq \frac{\pi}{2}-\frac{\alpha}{2} .
$$

If $e^{i \phi}$ is an interior point of an arc which is mapped by $h(\zeta)$ onto an arc of $|z|=1$, then $\psi(\phi)=0$. At every point $z_{0}=h\left(e^{i \phi_{0}}\right)$ of the boundary $\gamma$ of $\Gamma$, with $\left|z_{0}\right|=1$, a chord $z_{0} z$ of $\gamma$ forms an angle $\chi\left(z_{0}, z\right)$ with the tangent line to the unit circle at $z_{0}$ such that $\lim \sup _{z \rightarrow z_{0}}\left|\chi\left(z_{0}, z\right)\right| \leqq \pi / 2-\alpha / 2$. Hence, for $|\zeta|=1$, $\zeta_{0}=e^{i \phi_{0}}$,

$$
\limsup _{\zeta \rightarrow \zeta_{0}}\left|\arg \left[\frac{h(\zeta)-h\left(\zeta_{0}\right)}{\zeta-\zeta_{0}} \frac{\zeta}{h(\zeta)}\right]\right| \leqq \frac{\pi}{2}-\frac{\alpha}{2},
$$

and this holds also as $\zeta \rightarrow \zeta_{0}$ from $|\zeta|<1$, as is seen by use of the Poisson integral. But, if $\psi\left(\phi_{0}\right)$ exists,

$$
\lim _{\rho \rightarrow 1} \arg \left[\frac{h\left(\rho \zeta_{0}\right)-h\left(\zeta_{0}\right)}{(\rho-1) \zeta_{0}} \frac{\rho \zeta_{0}}{h\left(\rho \zeta_{0}\right)}\right]=\lim _{\rho \rightarrow 1} \arg \frac{\rho \zeta_{0} h^{\prime}\left(\rho \zeta_{0}\right)}{h\left(\rho \zeta_{0}\right)}=\psi\left(\phi_{0}\right),
$$

and thus $\left|\psi\left(\phi_{0}\right)\right| \leqq \pi / 2-\alpha / 2$. Hence, wherever $\psi(\phi)$ is defined,

$$
|\psi(\phi)| \leqq \frac{\pi}{2}-\frac{\alpha}{2} .
$$

Because of (2.13), $V(\zeta)$ may be represented by the Poisson integral in $|\zeta|<1$ with the boundary values $\psi(\phi)$ and hence

$$
|V(\zeta)| \leqq \frac{\pi}{2}-\frac{\alpha}{2}, \text { for }|\zeta|<1
$$

By a lemma of Zygmund [15] we have, therefore, for $\zeta=\rho e^{i \phi}$,

$$
\mathfrak{M}_{q}\left\{\left|\zeta \frac{h^{\prime}(\zeta)}{h(\zeta)}\right|^{ \pm 1}\right\} \leqq\left[\frac{2}{\cos q(\pi / 2-\alpha / 2)}\right]^{1 / q}, \quad 0<\rho \leqq 1,
$$

for any $q>0$ such that $q(\pi / 2-\alpha / 2)<\pi / 2$.

(c) Let $z=r(\theta) e^{i \theta}$ be a point on the boundary of $\Gamma$. The arc $w=f\left(\rho e^{i \theta}\right)$, $r(\theta) \leqq \rho \leqq 1$, has the length

$$
\lambda_{\theta}=\int_{r(\theta)}^{1}\left|f^{\prime}\left(\rho e^{i \theta}\right)\right| d \rho \geqq(1-r(\theta)) \operatorname{Inf}_{0 \leqq \rho<1}\left|f^{\prime}\left(\rho e^{i \theta}\right)\right| .
$$

Because of the hypothesis (iii) of $\S 1$ we have by Lemma $2, \lambda_{\theta} \leqq 2 \epsilon$ and, therefore, 


$$
1-r(\theta) \leqq 2 \epsilon g(\theta), \quad g(\theta)=\operatorname{Sup}_{0 \leqq \rho<1} \frac{1}{\left|f^{\prime}\left(\rho e^{i \theta}\right)\right|} .
$$

By a theorem of Hardy and Littlewood [3], there exists a constant $L_{p}$, which depends only on $p$, such that

$$
\mathfrak{M}_{p}\{g(\theta)\} \leqq L_{p} \mathfrak{M}_{p}\left\{\frac{1}{\left|f^{\prime}\left(e^{i \theta}\right)\right|}\right\} \leqq L_{p} N_{p}
$$

and $N_{p}$ depends only on $p, d, D, c$, and $\beta(t)$ by [12]. We have, therefore,

$$
\mathfrak{M}_{p}\{1-r(\theta)\} \leqq 2 \epsilon \mathfrak{M}_{p}\{g(\theta)\} \leqq 2 \epsilon L_{p} N_{p} .
$$

Since $C_{1}$ lies exterior to $|w|=d$, we have by Lemma $1, r(\theta) \geqq D / d$ and thus

$$
|\log r(\theta)| \leqq \frac{1-r(\theta)}{r(\theta)} \leqq \frac{D}{d}(1-r(\theta)) .
$$

We have, therefore,

$$
\mathfrak{M}_{p}\{\log r(\theta)\} \leqq \frac{D}{d} L_{p} N_{p} 2 \epsilon .
$$

The relation $r(\theta) e^{i \theta}=h\left(e^{i \phi}\right)$ defines $\theta$ as a monotone and continuous function of $\phi, \theta=\theta(\phi)$, and because of $(2.8), \theta(\phi)$ is even absolutely continuous. Hence

$$
\begin{aligned}
\left\{\frac{1}{2 \pi} \int_{0}^{2 \pi}|\log r(\theta)| p d \phi\right\}^{1 / p} & \\
& =\left\{\frac{1}{2 \pi} \int_{0}^{2 \pi}|\log r(\theta)|^{p}\left(\frac{d \theta}{d \phi}\right)^{1 / 2}\left(\frac{d \theta}{d \phi}\right)^{-1 / 2} d \phi\right\}^{1 / p} \\
& \leqq\left\{\frac{1}{2 \pi} \int_{0}^{2 \pi}|\log r(\theta)|^{2 p} \frac{d \theta}{d \phi} d \phi\right\}^{1 / 2 p}\left\{\frac{1}{2 \pi} \int_{0}^{2 \pi} \frac{d \phi}{d \theta / d \phi}\right\}^{1 / 2 p} .
\end{aligned}
$$

It is easily seen that $d \theta / d \phi \geqq \cos (\pi / 2-\alpha / 2)\left|h^{\prime}\left(e^{i \phi}\right) / h\left(e^{i \phi}\right)\right|$ almost everywhere, and hence, by (2.8), for $q=1$,

$$
\mathfrak{M}_{1}\left\{\left(\frac{d \theta}{d \phi}\right)^{-1}\right\} \leqq \frac{2}{\sin ^{2}(\alpha / 2)} .
$$

Thus we have, for $\zeta=e^{i \phi}$,

$$
\mathfrak{M}_{p}\left\{\log \left|\frac{h(\zeta)}{\zeta}\right|\right\} \leqq 4 \epsilon \frac{D}{d \sin ^{2}(\alpha / 2)} L_{2 p} N_{2 p},
$$

and by the well known theorem of M. Riesz on conjugate functions [5]

$$
\mathfrak{M}_{p}\left\{\log \frac{h(\zeta)}{\zeta}\right\} \leqq\left(1+A_{p}\right) \frac{4 D \epsilon}{d \sin ^{2}(\alpha / 2)} L_{2 p} N_{2 p}=H_{p} \epsilon
$$


where $A_{p}$ depends only on $p$. Hence

$$
\mathfrak{M}_{p}\left\{\frac{h(\zeta)}{\zeta}-1\right\} \leqq H_{p} \epsilon
$$

which implies (2.9).

3. Proof of Theorem I, Part (1). We assume first that $\epsilon \leqq \epsilon_{1}$, the bound of Lemma 3. Suppose $G, \Gamma$, and $h(\zeta)$ have the same meaning as in Lemma 3. Then $g(\zeta)=f(h(\zeta))$ maps $|\zeta|<1$ onto $G$ such that $g(0)=0, g^{\prime}(0)>0$. If the boundary of $G$ is given by the equation $r=r(\theta)$, and if $\arg h\left(e^{i \phi}\right)=\theta(\phi)$, we have, for $\zeta=e^{i \phi}$,

$$
\begin{aligned}
\mathfrak{M}_{p}\{g(\zeta)-f(\zeta)\} \leqq & \left\{\frac{1}{2 \pi} \int_{0}^{2 \pi}\left|f\left(r(\theta) e^{i \theta}\right)-f\left(e^{i \theta}\right)\right| p d \phi\right\}^{1 / p} \\
& +\mathfrak{M}_{p}\left\{f\left(e^{i \theta(\phi)}\right)-f\left(e^{i \phi}\right)\right\}=I_{1}+I_{2}
\end{aligned}
$$

By Hypothesis (iii) and Lemma $2, I_{1} \leqq 2 \epsilon$.

To estimate $I_{2}$ we write $s^{\prime}(t)=\left|f^{\prime}\left(e^{i t}\right)\right|$ and obtain

$$
I_{2} \leqq\left\{\frac{1}{2 \pi} \int_{0}^{2 \pi}\left|\int_{\phi}^{\theta(\phi)} s^{\prime}(t) d t\right|^{p} d \phi\right\}^{1 / p} .
$$

Since $(1 / 2 \pi) \int_{0}^{2 \pi}(\theta(\phi)-\phi) d \phi=\arg h^{\prime}(0)=0$ there exists a value $a, 0 \leqq a \leqq 2 \pi$, such that $\theta(a)-a=0$. Using the periodicity of the integrand we may replace the limits 0 and $2 \pi$ in (3.1) by $a$ and $2 \pi+a$. By Hölder's inequality:

$$
\begin{aligned}
I_{2} & \leqq\left\{\frac{1}{2 \pi} \int_{a}^{2 \pi+a} \frac{1}{\theta(\phi)-\phi} \int_{\phi}^{\theta(\phi)}\left(s^{\prime}(t)\right)^{p} d t|\theta(\phi)-\phi|^{p} d \phi\right\}^{1 / p} \\
& \leqq\left\{\frac{1}{2 \pi} \int_{a}^{2 \pi+a} F(\phi)|\theta(\phi)-\phi|^{p} d \phi\right\}^{1 / p}
\end{aligned}
$$

where

$$
F(\phi)=\operatorname{Sup}_{a \leqq \xi \leqq 2 \pi+a} \frac{1}{\xi-\phi} \int_{\phi}^{\xi}\left[s^{\prime}(t)\right]^{p} d t
$$

(note that $a \leqq \phi, \theta(\phi) \leqq 2 \pi+a)$. Applying the inequality of Schwarz we obtain

$$
I_{2} \leqq\left(\mathfrak{M}_{2}\{F(\phi)\}\right)^{1 / p \mathfrak{M}_{2 p}}\{\theta(\phi)-\phi\} .
$$

By a theorem of Hardy and Littlewood [3], see also [16, p. 244]:

$$
\left[\mathfrak{M}_{2}\{F(\phi)\}\right]^{1 / p} \leqq 2\left(2^{1 / 2}\right)\left[\mathfrak{M}_{2}\left\{\left(s^{\prime}(t)\right)^{p}\right\}\right]^{1 / p}=2\left(2^{1 / 2}\right) N_{2 p}
$$

by [12]. Hence, by Lemma 3, (2.9), and Lemma 1 ,

$$
I_{2} \leqq 2\left(2^{1 / 2}\right) N_{2 p} \mathfrak{M}_{2 p}\{\theta(\phi)-\phi\} \leqq D_{p} \epsilon,
$$

where $D_{p}$ depends only on $p, d, D, c, \alpha, \beta$, and $\beta_{1}$. We have thus 


$$
\mathfrak{M}_{p}\{f-g\} \leqq M_{p}^{\prime} \epsilon
$$

where $M_{p}^{\prime}$ depends only on these seven parameters. This inequality was proved under the assumption $\epsilon \leqq \epsilon_{1}$. However, if $\epsilon>\epsilon_{1}$ we write $\mathfrak{M}_{p}\{f-g\}$ $\leqq 2 D \leqq\left(2 D / \epsilon_{1}\right) \epsilon$ and thus (3.2) is true for all $\epsilon>0$.

Using Remark 1 on the condition ( $v)$ of $\$ 1$ and applying an analogous argument to $f_{1}(z)$ and $g(z)$ we obtain $\left(\zeta=e^{r \phi}\right)$

$$
\mathfrak{M}_{p}\left\{f_{1}(\zeta)-g(\zeta)\right\} \leqq M_{p}^{\prime \prime} \epsilon,
$$

where $M_{p}^{\prime \prime}$ depends on the same parameters as $M_{p}^{\prime}$. The conclusion (1.2) follows now by combination of (3.2) and (3.3).

4. Proof of Theorem I, Part (2). Again, it is sufficient to prove the conclusion assuming $\epsilon \leqq \epsilon_{2}$, where $\epsilon_{2}$ depends only on $p, d, D, c, \alpha$, and the functions $\beta$ and $\beta_{1}$. For if $\epsilon>\epsilon_{2}$ we have by $[12$, p. 254],

$$
\mathfrak{M}_{p}\left\{f^{\prime}-f_{1}^{\prime}\right\} \leqq \frac{2 B_{p}}{\beta\left(\epsilon_{2}\right)} \beta(\epsilon) \leqq \frac{2 B_{p}}{\beta\left(\epsilon_{2}\right)}[\eta+\beta(\epsilon)] \leqq \frac{2 B_{p}}{\beta\left(\epsilon_{2}\right)}\left(\eta+\beta^{*}(\epsilon)\right) .
$$

(We may assume $M_{p} \geqq 1$.)

In accordance with (2.1) we have

$$
\arg f_{1}^{\prime}\left(e^{i \theta}\right)-\arg f^{\prime}\left(e^{i \theta}\right)=\tau_{1}\left[s_{1}(\theta)\right]-\tau[s(\theta)] .
$$

By hypothesis (v) of $\S 1$ there exists for every $s_{1}(\theta)$ a value $\sigma=s\left(\theta_{1}\right)$ such that $\left|f\left(e^{i \theta 1}\right)-f_{1}\left(e^{i \theta}\right)\right| \leqq \epsilon$ and, for a suitable branch of $\tau(\sigma)$,

$$
\left|\tau_{1}\left[s_{1}(\theta)\right]-\tau(\sigma)\right| \leqq \eta \text {. }
$$

We may choose $\sigma$ so that $|s(\theta)-\sigma| \leqq L / 2$. Then, for a suitable integer $k$, which may depend on $\theta$,

$$
|\tau(\sigma)-\tau[s(\theta)]+2 k \pi| \leqq \beta(|\sigma-s(\theta)|) \leqq \beta\left(c\left|f\left(e^{i \theta_{1}}\right)-f\left(e^{i \theta}\right)\right|\right),
$$

by hypothesis (ii). Since $\left|f\left(e^{i \theta_{1}}\right)-f\left(e^{i \theta}\right)\right| \leqq \epsilon+\left|f_{1}\left(e^{i \theta}\right)-f\left(e^{i \theta}\right)\right|$,

$$
|\tau(\sigma)-\tau[s(\theta)]+2 k \pi| \leqq \beta\left(c\left[\epsilon+\left|f_{1}\left(e^{i \theta}\right)-f\left(e^{i \theta}\right)\right|\right]\right) .
$$

Hence

$$
\left|\arg f_{1}^{\prime}\left(e^{i \theta}\right)-\arg f^{\prime}\left(e^{i \theta}\right)+2 k \pi\right| \leqq \eta+\beta\left(c\left[\epsilon+\left|f_{1}\left(e^{i \theta}\right)-f\left(e^{i \theta}\right)\right|\right]\right) .
$$

We maintain now that $k=0$, if $\epsilon$ is sufficiently small. We show first that $k$ is independent of $\theta$. By [11, Theorem VIII], there exists a constant $m$, which depends only on $d, D, c$ and the functions $\beta, \beta_{1}$ such that

$$
\left|f_{1}\left(e^{i \theta}\right)-f\left(e^{i \theta}\right)\right| \leqq m\left(\epsilon^{1 / 2}\right) .
$$

Hence we can determine an $\epsilon_{2}>0$ which depends only on the just mentioned parameters such that, for $\epsilon \leqq \epsilon_{2}$, the right-hand side of (4.2) does not exceed $\pi / 2$. Now, $\tau[s(\theta)]$ is a continuous function of $\theta ; \tau(\sigma)$, considered as a function 
of $\theta$, may possibly not be continuous, but, because of the continuity of $\tau_{1}\left[s_{1}(\theta)\right]$ and of (4.1), there exists an $\omega>0$ such that the oscillation of $\tau(\sigma)$ in any sub-interval of $0 \leqq \theta \leqq 2 \pi$ of length $\leqq \omega$ is less than $2 \eta+\alpha \leqq \pi-\alpha$. Hence, there exists an $\omega_{1}>0$ such that the oscillation of $\tau(\sigma)-\tau[s(\theta)]$ in any interval of length $\leqq \omega_{1}$ is less than $\pi$. If, for some $\theta, k$ changed as $\theta$ varied from $\theta$ to $\theta+\Delta \theta\left(|\Delta \theta| \leqq \omega_{1}\right)$, the left-hand side of (4.2) would change by an amount whose modulus is $>\pi$, which contradicts the fact that it is and must remain $\leqq \pi / 2$. By employing a partition of $[0,2 \pi]$ with norm $\leqq \omega_{1}$, we see that $k$ is constant for all $\theta, 0 \leqq \theta \leqq 2 \pi$.

We apply now the maximum and minimum principles to $2 \pi k+\arg f_{1}^{\prime}(z)$ $-\arg f^{\prime}(z)$. Because of (4.3) and of our choice $\epsilon \leqq \epsilon_{2}$ we have, for $z=0$,

$$
|2 \pi k|=\eta \leqq \frac{\pi}{2}<\frac{3}{2} \pi,
$$

which implies $k=0$.

We obtain now from (4.3) with $k=0$

$$
\mathfrak{M}_{p}\left\{\arg f_{1}^{\prime}\left(e^{i \theta}\right)-\arg f^{\prime}\left(e^{i \theta}\right)\right\} \leqq \eta+\mathfrak{M}_{p}\left\{\beta\left(c\left[\epsilon+\left|f_{1}\left(e^{i \theta}\right)-f\left(e^{i \theta}\right)\right|\right]\right)\right\}
$$

and if $\beta$ is replaced by the convex majorant $\beta^{*}$, we have, using (1.2)

$\mathfrak{M}_{p}\left\{\arg f_{1}^{\prime}\left(e^{i \theta}\right)-\arg f^{\prime}\left(e^{i \theta}\right)\right\} \leqq \eta+\beta^{*}\left(c \epsilon+M_{p} c \epsilon\right) \leqq \eta+\beta^{*}\left(2 c M_{p} \epsilon\right)$.

Hence, by the theorem of M. Riesz on conjugate functions [5],

$$
\begin{aligned}
\mathfrak{M}_{p}\left\{\log f_{1}^{\prime}\left(e^{i \theta}\right)-\log f^{\prime}\left(e^{i \theta}\right)\right\} \leqq & \left(1+A_{p}\right)\left(\eta+\beta^{*}\left(2 c M_{p} \epsilon\right)\right) \\
& +\left|\log \frac{f_{1}^{\prime}(0)}{f^{\prime}(0)}\right| .
\end{aligned}
$$

Noting that $f_{1}^{\prime}(0) \geqq d, f^{\prime}(0) \geqq d$, we obtain by $(1.2)$

$$
\begin{aligned}
\left|\log f_{1}^{\prime}(0)-\log f^{\prime}(0)\right| & \leqq \frac{1}{d}\left|f_{1}^{\prime}(0)-f^{\prime}(0)\right| \leqq \frac{1}{d} M_{p} \epsilon \\
& \leqq \frac{M_{p}}{d k_{0}} \beta^{*}\left(2 c M_{p} \epsilon\right),
\end{aligned}
$$

where $k_{0}=\beta\left(\epsilon_{2}\right) / \epsilon_{2}\left({ }^{6}\right)$. Since

$$
\left|f^{\prime}-f_{1}^{\prime}\right| \leqq\left(\left|f^{\prime}\right|+\left|f_{1}^{\prime}\right|\right)\left|\log f^{\prime}-\log f_{1}^{\prime}\right|
$$

we obtain by use of the inequalities of Schwarz and Minkowski

$$
\mathfrak{M}_{p}\left\{f^{\prime}-f_{1}^{\prime}\right\} \leqq\left[\mathfrak{M}_{2 p}\left\{f_{1}^{\prime}\right\}+\mathfrak{M}_{2 p}\left\{f^{\prime}\right\}\right] \mathfrak{M}_{2 p}\left\{\log f^{\prime}-\log f_{1}^{\prime}\right\} .
$$

By $[12$, p. 254], and by (4.4) and (4.5) we have finally

$$
\mathfrak{M}_{p}\left\{f^{\prime}-f_{1}^{\prime}\right\} \leqq K_{p}\left(\eta+\beta^{*}\left(2 c M_{p} \epsilon\right)\right) \text {. }
$$

$\left.{ }^{(}\right)$Since $\beta^{*}(\epsilon)$ is convex from above we have, for $0<\epsilon \leqq \epsilon_{2}, \beta^{*}(\epsilon) / \epsilon \geqq \beta^{*}\left(\epsilon_{2}\right) / \epsilon_{2} \geqq \beta(\epsilon) / \epsilon$. 


\section{UNIFORM ESTIMATES}

5. An estimate for the difference of the mapping functions. We retain the notations of $\S 1$ and derive here estimates for

$$
\mu=\operatorname{Sup}_{|z| \leqq 1}\left|f(z)-f_{1}(z)\right| \quad \text { and } \quad \mu^{\prime}=\operatorname{Sup}_{|z| \leqq 1}\left|f^{\prime}(z)-\right| f_{1}^{\prime}(z) \mid .
$$

An immediate bound for $\mu$ is obtained from (1.2) by use of the FejérRiesz inequality (see $[1$, Satz II $]$ ). We shall prove here yet another result. Let, for $t>0$,

$$
\omega(t)=\operatorname{Max}\left\{\int_{0}^{t} \frac{\beta(x)}{x} d x, \int_{0}^{t} \frac{\beta_{1}(x)}{x} d x\right\}
$$

Then we have the following

Theorem II. Suppose that $C$ and $C_{1}$ satisfy the hypotheses (i), (ii), (iii) and (iv). Suppose furthermore that $\omega(t)<\infty$ for finite $t$. Then there exists a constant $M$ which depends only on $d, D, c$ and $\beta(t)$ and $\beta_{1}(t)$ such that $\mu \leqq M \epsilon \log 1 / \epsilon$.

The proof is modeled after that of Theorem VIII of [11]. Since $\omega(t)<\infty$ there exist two constants $a_{1}, a_{2}$ which depend only on the parameters named above such that $\left({ }^{7}\right)$, for $|z| \leqq 1,\left|z_{0}\right|=1$,

$$
a_{1}\left|z-z_{0}\right| \leqq\left|f(z)-f\left(z_{0}\right)\right| \leqq a_{2}\left|z-z_{0}\right| \text {. }
$$

If these inequalities are used in place of (6.2) of [11], the proof on pp. 354355 yields the conclusion of the theorem.

6. Estimates for $\mu^{\prime}$. We turn now to the consideration of $\mu^{\prime}$ and derive first a general result from which simpler statements may be obtained by choosing special forms for the parameters which characterize the geometrical configuration.

Theorem III. Suppose that $C$ and $C_{1}$ satisfy hypotheses (i)-(v) of $\$ 1$ and that $\omega(t)<\infty$ for $t<\infty$. Let

$$
\lambda=\operatorname{Max}_{\mid z i \leqq 1}\left\{\left|f^{\prime}(z)\right|,\left|f_{1}^{\prime}(z)\right|\right\} .
$$

( $A$ bound for $\lambda$ exists which depends only on $d, D, c$ and the functions $\beta$ and $\beta_{1}\left({ }^{8}\right)$ ). Then, for every $\delta, 0<\delta<\pi$,

$$
\mu^{\prime} \leqq \frac{2 \lambda}{\pi}\left[\omega(2 \lambda \delta)+\{\eta+\beta(c[\epsilon+\mu])\}\left(\frac{\pi}{2}+\log \frac{\pi}{\delta}\right)\right]+\frac{\mu}{d}
$$

provided $\beta(c[\epsilon+\mu]) \leqq \pi / 2$. In particular, $\delta$ may be chosen as a function of $\epsilon$.

(7) This follows from $[9$, p. 440] and Lemma 10 of that paper on p. 376.

$\left.{ }^{8}\right)$ The existence of such a bound follows from $[10$, p. 440]; see also [10, p. 327]. 
Proof. Let $\log f^{\prime}(z)$ and $\log f_{1}^{\prime}(z)$ denote the branches of the logarithms which are real at $z=0$. These are single-valued and continuous in $|z| \leqq 1$. Let

$$
A(\theta, t)=\arg f^{\prime}\left(e^{i(\theta+t)}\right)-\arg f^{\prime}\left(e^{i(\theta-t)}\right)
$$

and let $A_{1}(\theta, t)$ denote the same expression formed for $f_{1}(z)$. Then

$$
\begin{aligned}
\log \left|\frac{f^{\prime}\left(e^{i \theta}\right)}{f^{\prime}(0)}\right|- & \log \left|\frac{f_{1}^{\prime}\left(e^{i \theta}\right)}{f_{1}^{\prime}(0)}\right| \\
= & \frac{1}{2 \pi} \int_{0}^{\pi}\left[A(\theta, t)-A_{1}(\theta, t)\right] \cot \frac{t}{2} d t \\
= & \frac{1}{2 \pi} \int_{0}^{\delta}(A(\theta, t)+2 t) \cot \frac{t}{2} d t-\frac{1}{2 \pi} \int_{0}^{\delta}\left(A_{1}(\theta, t)+2 t\right) \cot \frac{t}{2} d t \\
& +\frac{1}{2 \pi} \int_{\delta}^{\pi}\left[A(\theta, t)-A_{1}(\theta, t)\right] \cot \frac{t}{2} d t=I_{1}-I_{2}+I_{3} .
\end{aligned}
$$

By (2.1), (2.2), and (6.1),

$$
|A(\theta, t)+2 t| \leqq \beta(|s(\theta+t)-s(\theta-t)|) \leqq \beta(2 \lambda t)
$$

and therefore

$$
\left|I_{1}\right| \leqq \frac{1}{2 \pi} \int_{0}^{\delta} \frac{\beta(2 \lambda t)}{t / 2} d t \leqq \frac{1}{\pi} \omega(2 \lambda \delta) .
$$

Similarly

$$
\left|I_{2}\right| \leqq \frac{1}{\pi} \omega(2 \lambda \delta) .
$$

To estimate $I_{3}$ we apply (4.3), noting that $\beta(c[\epsilon+\mu]) \leqq \pi / 2$ and hence $k=0$, and obtain

$$
\left|A(\theta, t)-A_{1}(\theta, t)\right| \leqq 2(\eta+\beta(c[\epsilon+\mu]) .
$$

Hence

$$
\left|I_{3}\right| \leqq 2(\eta+\beta(c[\epsilon+\mu])) \frac{1}{\pi} \int_{\delta}^{\pi} \frac{d t}{t}=\frac{2}{\pi}[\eta+\beta(c[\epsilon+\mu])] \log \frac{\pi}{\delta} .
$$

Thus we obtain

$\left|\log f^{\prime}\left(e^{i \theta}\right)-\log f_{1}^{\prime}\left(e^{i \theta}\right)\right|$

$$
\begin{aligned}
& \leqq|\log | f^{\prime}\left(e^{i \theta}\right)|-\log | f_{1}^{\prime}\left(e^{i \theta}\right)||+\left|\arg f^{\prime}\left(e^{i \theta}\right)-\arg f_{1}^{\prime}\left(e^{i \theta}\right)\right| \\
& \leqq \frac{2}{\pi} \omega(2 \lambda \delta)+\frac{2}{\pi}(\eta+\beta(c[\epsilon+\mu])) \log \frac{\pi}{\delta}+\eta+\beta(c[\epsilon+\mu])+\left|\log \frac{f^{\prime}(0)}{f_{1}^{\prime}(0)}\right| .
\end{aligned}
$$


Here $\left|\log \left(f^{\prime}(0) / f_{1}^{\prime}(0)\right)\right| \leqq \mu / d$. Finally, using the inequality

$$
\left|f^{\prime}(z)-f_{1}^{\prime}(z)\right| \leqq \lambda\left|\log f^{\prime}(z)-\log f_{1}^{\prime}(z)\right|
$$

where $\lambda$ is defined by (6.1), we obtain (6.2).

As an example of a simple result which follows from (6.2) we state

Theorem IV. If $C$ and $C_{1}$ satisfy hypotheses (i)-(v) and if $\beta(t)=H t$, $\beta_{1}(t)=H_{1} t^{\gamma}, 0<\gamma \leqq 1, \eta \leqq q \epsilon$, where $H, H_{1}, \gamma$, and $q$ are constants, then

$$
\mu \leqq M \epsilon \text { and } \mu^{\prime} \leqq K \epsilon \log \frac{\pi}{\epsilon}
$$

where $M$ and $K$ depend only on $d, D, c, H, H_{1}, \gamma$, and $q$.

Proof. From (1.3) we obtain by the Fejér-Riesz inequality, for $p=2$,

$$
\mu \leqq \pi K_{2}\left(\eta+\beta^{*}\left(2 c M_{2} \epsilon\right)\right)=\pi K_{2}\left(q \epsilon+H 2 c M_{2} \epsilon\right)=M \epsilon,
$$

since $\beta(t)=H t$ may be taken as $\beta^{*}(t)$.

Next we note that it is sufficient to prove the inequality concerning $\mu^{\prime}$ under the assumption $\epsilon \leqq \pi / 2 H c(1+M)=\epsilon_{0}$; this insures that $\beta(c[\epsilon+\mu]) \leqq \pi / 2$ for $\epsilon \leqq \epsilon_{0}$. If $\epsilon>\epsilon_{0}$, then $\mu^{\prime} \leqq 2 \lambda_{0} \epsilon / \epsilon_{0}$, where $\lambda_{0}$ is an upper bound for $\lambda$ (see (6.1)) and depends only on the seven constants named in the theorem. Choosing $\delta=\epsilon^{1 / \gamma}$ we obtain from (6.2) (assuming $\gamma H \leqq H_{1}$ )

$$
\mu^{\prime} \leqq \frac{2 \lambda}{\pi}\left[\frac{H_{1}}{\gamma}(2 \lambda)^{\gamma} \epsilon+(q \epsilon+H c[\epsilon+M \epsilon])\left(\frac{\pi}{2}+\log \frac{\pi}{\epsilon^{1 / \gamma}}\right)\right]+\frac{\mu}{d} \leqq K \epsilon \log \frac{\pi}{\epsilon} .
$$

7. Derivatives of the inverse functions. In order to state our next result it will be convenient to assume that $C$ and $C_{1}$ are represented in parametric form by the equations

$$
w=w(t) \text { and } w=w_{1}(t),
$$$$
0 \leqq t \leqq 1,
$$

respectively; $w(t), w_{1}(t)$ have the period 1 and $w^{\prime}(t)$ and $w_{1}^{\prime}(t)$ have bounded difference quotients; thus $w^{\prime \prime}(t)$ and $w_{1}^{\prime \prime}(t)$ exist almost everywhere in $[0,1]$ and

$$
\left|w^{\prime \prime}(t)\right| \leqq k, \quad\left|w_{1}^{\prime \prime}(t)\right| \leqq k
$$

where $k$ is a constant. We assume furthermore that

$$
\left|w^{\prime}(t)\right| \geqq b>0, \quad\left|w_{1}^{\prime}(t)\right| \geqq b>0,
$$

and that for some $\epsilon>0$ and some $p>1$

$$
\mathfrak{M}_{p}\left\{w^{\prime \prime}(t)-w_{1}^{\prime \prime}(t)\right\} \leqq \epsilon \text { and }\left|w(0)-w_{1}(0)\right| \leqq \epsilon .
$$

Theоrem V. Suppose $C$ and $C_{1}$ satisfy hypotheses (i) and (ii) of $\S 1$ and are represented by the functions (7.1) with the properties (7.2), (7.3), (7.4). Let 
$\phi(w)$ and $\phi_{1}(w)$ be the inverse functions of $f(z)$ and $f_{1}(z)$, respectively. Then there exists a constant $N$ which depends only on $d, D, c, b, k$ and $p$ such that

$$
\operatorname{Sup}_{0 \leqq t \leqq 1}\left|\phi^{\prime}(w(t))-\phi_{1}^{\prime}\left(w_{1}(t)\right)\right| \leqq N \epsilon .
$$

Before passing on to the proof we mention several consequences of the above hypotheses.

1. Since $\int_{0}^{1}\left(w^{\prime}(t)-w_{1}^{\prime}(t)\right) d t=0$ there exist values $t_{0}$ and $t_{1}, 0 \leqq t_{0}, t_{1} \leqq 1$, such that $\operatorname{Re}\left[w^{\prime}\left(t_{0}\right)-w_{1}^{\prime}\left(t_{0}\right)\right]=0$ and $\operatorname{Im}\left[w^{\prime}\left(t_{1}\right)-w_{1}^{\prime}\left(t_{1}\right)\right]=0$. Since, for $0 \leqq t \leqq 1$,

$$
\begin{gathered}
\operatorname{Re}\left[w^{\prime}(t)-w_{1}^{\prime}(t)\right]=\int_{t_{0}}^{t} \operatorname{Re}\left[w^{\prime \prime}(\xi)-w_{1}^{\prime \prime}(\xi)\right] d \xi, \\
\operatorname{Im}\left[w^{\prime}(t)-w_{1}^{\prime}(t)\right]=\int_{t_{1}}^{t} \operatorname{Im}\left[w^{\prime \prime}(\xi)-w_{1}^{\prime \prime}(\xi)\right] d \xi, \\
\left|w^{\prime}(t)-w_{1}^{\prime}(t)\right| \leqq 2 \mathfrak{M}_{p}\left\{w^{\prime \prime}-w_{1}^{\prime \prime}\right\} \leqq 2 \epsilon .
\end{gathered}
$$

2. From the second inequality in (7.4) and from (7.6)

$$
\left|w(t)-w_{1}(t)\right| \leqq 3 \epsilon .
$$

3. Let $\tau(t)$ denote the tangent angle to $C$ at the point $w(t)$. Then $\kappa(t)$ $=d \tau / d t=\operatorname{Im}\left[w^{\prime \prime} / w^{\prime}\right]$ for almost all $t$. If $\tau_{1}(t), \kappa_{1}(t)$ have the analogous meaning for $C_{1}$,

$$
\begin{aligned}
\mathfrak{M}_{p}\left\{\kappa(t)-\kappa_{1}(t)\right\} & \leqq \frac{1}{b} \mathfrak{M}_{p}\left\{w^{\prime \prime}-w_{1}^{\prime \prime}\right\}+\frac{k}{b^{2}} \mathfrak{M}_{p}\left\{w^{\prime}-w_{1}^{\prime}\right\} \\
& \leqq \frac{b+2 k}{b^{2}} \epsilon .
\end{aligned}
$$

Furthermore, $\left|\tau\left(t_{2}\right)-\tau\left(t_{1}\right)\right| \leqq(k / b)\left|t_{2}-t_{1}\right|$ and we may take $\beta(s)=\left(k / b^{2}\right) s$ and similarly $\beta_{1}(s)=\left(k / b^{2}\right) s$.

Since $\int_{0}^{1} w^{\prime}(t) d t=\int_{0}^{1} w_{1}^{\prime}(t) d t=0$, it follows from (7.2) that

$$
\left|w^{\prime}(t)\right| \leqq 2 k, \quad\left|w_{1}^{\prime}(t)\right| \leqq 2 k .
$$

8. Proof of Theorem V. (a) An integral representation for

$$
\log \left|\phi^{\prime}(w(t)) / \phi_{1}^{\prime}\left(w_{1}(t)\right)\right| \text {. }
$$

We have by an integration by parts

$$
\begin{aligned}
\log \left|f^{\prime}\left(e^{i \theta_{0}}\right)\right| & =\frac{1}{2 \pi} \int_{\theta_{0}-\pi}^{\theta_{0}+\pi}\left[\arg f^{\prime}\left(e^{i \theta}\right)-\arg f^{\prime}\left(e^{i \theta_{0}}\right)\right] \cot \frac{\theta-\theta_{0}}{2} d \theta+\log f^{\prime}(0) \\
& =-\frac{1}{\pi} \int_{\theta_{0}-\pi}^{\theta_{0}+\pi} \frac{d \arg f^{\prime}\left(e^{i \theta}\right)}{d \theta} \log \left|\sin \frac{\theta-\theta_{0}}{2}\right| d \theta+\log f^{\prime}(0)
\end{aligned}
$$


The function $\theta(t)=\arg \phi(w(t))$ is (strictly) monotone and continuously differentiable. Let $t(\theta)$ denote its inverse function. If $t_{0}=t\left(\theta_{0}\right)$

$$
\begin{aligned}
\log \left|\phi^{\prime}\left(w\left(t_{0}\right)\right)\right|= & \frac{1}{\pi} \int_{0}^{2 \pi}\left(\kappa(t) t^{\prime}(\theta)-1\right) \log \left|\sin \frac{\theta-\theta_{0}}{2}\right| d \theta+\log \phi^{\prime}(0) \\
= & \frac{1}{\pi} \int_{0}^{1} \kappa(t) \log \left|\sin \frac{\theta(t)-\theta\left(t_{0}\right)}{2}\right| d t-\frac{2}{\pi} \int_{0}^{\pi} \log \left|\sin \frac{t}{2}\right| d t \\
& +\log \phi^{\prime}(0) .
\end{aligned}
$$

Similarly, if $\theta_{1}(t)=\arg \phi_{1}\left(w_{1}(t)\right)$, we have

$$
\begin{aligned}
\log \left|\phi_{1}^{\prime}\left(w_{1}\left(t_{0}\right)\right)\right|= & \frac{1}{\pi} \int_{0}^{1} \kappa_{1}(t) \log \left|\sin \frac{\theta_{1}(t)-\theta_{1}\left(t_{0}\right)}{2}\right| d t \\
& -\frac{2}{\pi} \int_{0}^{\pi} \log \sin \frac{t}{2} d t+\log \phi_{1}^{\prime}(0) .
\end{aligned}
$$

\section{Hence}

$$
\begin{aligned}
\log \left|\frac{\phi^{\prime}\left(w\left(t_{0}\right)\right)}{\phi_{1}^{\prime}\left(w_{1}\left(t_{0}\right)\right)}\right|-\log \frac{\phi^{\prime}(0)}{\phi_{1}^{\prime}(0)}= & \frac{1}{\pi} \int_{0}^{1}\left[\kappa(t)-\kappa_{1}(t)\right] \log \left|\sin \frac{\theta(t)-\theta_{1}\left(t_{0}\right)}{2}\right| d t \\
& +\frac{1}{\pi} \int_{0}^{1} \kappa_{1}(t) \log \left|\frac{\sin \left(\left(\theta(t)-\theta\left(t_{0}\right)\right) / 2\right)}{\sin \left(\left(\theta_{1}(t)-\theta_{1}\left(t_{0}\right)\right) / 2\right)}\right| d t \\
= & I_{1}+I_{2} .
\end{aligned}
$$

(b) Estimate of $I_{1}$. The hypotheses (i), (ii) of $\$ 1,(7.3),(7.9)$ and the fact that $\beta(s)=\beta_{1}(s)=k s / b^{2}$ insure the existence of two positive constants, $a_{1}, a_{2}$, which depend only on $d, D, c, b$, and $k$ such that

$$
a_{1} \leqq \frac{d \theta}{d t} \leqq a_{2}, \quad a_{1} \leqq \frac{d \theta_{1}}{d t} \leqq a_{2} .
$$

By Hölder's inequality, (7.8), and (8.1) $(1 / p+1 / q=1)$

$$
\begin{aligned}
\left|I_{1}\right| & \leqq \frac{1}{\pi}\left[\int_{0}^{1}\left|\kappa(t)-\kappa_{1}(t)\right| p d t\right]^{1 / r}\left[\int_{0}^{1}|\log | \sin \frac{\theta(t)-\theta\left(t_{0}\right)}{2}||^{q} d t\right]^{1 / q} \\
& \leqq \frac{1}{\pi}\left[\int_{-\pi}^{\pi}|\log | \sin \frac{\theta-\theta_{0}}{2}||^{q} \frac{d t}{d \theta} d \theta\right]^{1 / q} \cdot \frac{b+2 k}{b^{2}} \epsilon \\
& \leqq \frac{(b+2 k) \epsilon}{\pi b^{2}}\left[\frac{2}{a_{1}} \int_{0}^{\pi}|\log | \sin \frac{x}{2}||^{q} d x\right]^{1 / q}=B_{0} \epsilon .
\end{aligned}
$$

(c) Estimate of $I_{2}$. We divide the interval into two parts. For some $\delta$, 
$0<\delta \leqq 1$, which will be specified below, let $E_{0}=[0,1] \cap\left[t_{0}-\delta, t_{0}+\delta\right]$ and $E_{1}$ the remainder of $[0,1]$. Let

$$
\begin{aligned}
j & =\log \left|\frac{\sin \left(\theta(t)-\theta\left(t_{0}\right)\right) / 2}{\sin \left(\theta_{1}(t)-\theta_{1}\left(t_{0}\right)\right) / 2}\right|=\log \left|1+\frac{\sin \left(\theta(t)-\theta\left(t_{0}\right)\right) / 2-\sin \left(\theta_{1}(t)-\theta_{1}\left(t_{0}\right)\right) / 2}{\sin \left(\theta_{1}(t)-\theta_{1}\left(t_{0}\right)\right) / 2}\right| \\
& =\log |1+u|,
\end{aligned}
$$

where

$$
u=\frac{2 \sin \left(\theta(t)-\theta\left(t_{0}\right)-\left[\theta_{1}(t)-\theta_{1}\left(t_{0}\right)\right] / 4\right)}{\left.\sin \left(\theta_{1}(t)-\theta_{1}\left(t_{0}\right)\right) / 2\right)} \cdot \cos \frac{\theta(t)-\theta\left(t_{0}\right)+\theta_{1}(t)-\theta_{1}\left(t_{0}\right)}{4} .
$$

By applying the (generalized) mean value theorem to the first factor we find, if $\delta a_{2} \leqq \pi / 2$, and $t_{0}-\delta \leqq t \leqq t_{0}+\delta$,

$$
|u| \leqq \frac{2^{1 / 2}}{\theta_{1}^{\prime}(\xi)}\left|\theta^{\prime}(\xi)-\theta_{1}^{\prime}(\xi)\right|, \quad\left|\xi-t_{0}\right|<\delta .
$$

Hence we have, for $t_{0}-\delta \leqq t \leqq t_{0}+\delta$, if $j \geqq 0$

$$
|j| \leqq \frac{2^{1 / 2}}{a_{1}} \operatorname{Max}_{0 \leqq t \leqq 1}\left|\theta^{\prime}(t)-\theta_{1}^{\prime}(t)\right| \text {. }
$$

If $j<0$, we consider $-j=\log \left(1+u^{\prime}\right)$ and find in the same manner

$$
\left|u^{\prime}\right| \leqq \frac{2^{1 / 2}}{\theta^{\prime}(\xi)}\left|\theta^{\prime}(\xi)-\theta_{1}^{\prime}(\xi)\right|, \quad\left|\xi-t_{0}\right|<\delta,
$$

and thus (8.3) holds also in this case.

For $t \in E_{1}$

$$
|u| \leqq \frac{\pi}{2 \alpha}\left\{\left|\theta(t)-\theta_{1}(t)\right|+\left|\theta_{1}\left(t_{0}\right)-\theta\left(t_{0}\right)\right|\right\}
$$

where $\alpha=\operatorname{Min}\left\{\left|\theta_{1}\left(t_{0}+\delta\right)-\theta_{1}\left(t_{0}\right)\right|,\left|\theta_{1}\left(t_{0}\right)-\theta_{1}\left(t_{0}-\delta\right)\right|\right\} \geqq \delta a_{1}$. Hence

$$
|u| \leqq \frac{\pi}{\delta a_{1}} \operatorname{Max}_{0 \leqq t \leqq 1}\left|\theta(t)-\theta_{1}(t)\right| .
$$

To estimate the last expression we write $\theta=\theta(t), \theta_{1}=\theta_{1}(t)$ and note that $\left|w(t)-w_{1}(t)\right|=\left|f\left(e^{i \theta}\right)-f_{1}\left(e^{i \theta_{1}}\right)\right| \geqq\left|f\left(e^{i \theta}\right)-f\left(e^{i \theta_{1}}\right)\right|-\left|f\left(e^{i \theta_{1}}\right)-f_{1}\left(e^{i \theta_{1}}\right)\right|$.

Hence,

$$
\begin{aligned}
\left|f\left(e^{i \theta}\right)-f\left(e^{i \theta_{1}}\right)\right| & \leqq\left|w(t)-w_{1}(t)\right|+\left|f\left(e^{i \theta_{1}}\right)-f_{1}\left(e^{i \theta_{1}}\right)\right| \\
& \leqq 3 \epsilon+M \epsilon=(3+M) \epsilon
\end{aligned}
$$

by (7.7) and Theorem IV. $M$ depends only on $d, D, c, k, b$. Since by (8.1) 


$$
\left|e^{i \theta}-e^{i \theta_{1}}\right| \leqq \frac{c a_{2}}{b}\left|f\left(e^{i \theta}\right)-f\left(e^{i \theta_{1}}\right)\right| \leqq \frac{c a_{2}}{b}(3+M) \epsilon
$$

we have, if $\left|\theta-\theta_{1}\right| \leqq \pi$,

$$
\left|\theta(t)-\theta_{1}(t)\right| \leqq \frac{2}{\pi}\left|e^{i \theta}-e^{i \theta_{1}}\right| \leqq \frac{2 c a_{2}}{\pi b}(3+M) \epsilon .
$$

Now, $\theta(t)$ and $\theta_{1}(t)$ are determined only up to an additive multiple of $2 \pi$. We choose the branch of $\theta(t)$ arbitrarily, say, such that $\theta(0)$ is the principal value, and then take $\theta_{1}(0)$ such that $\left|\theta(0)-\theta_{1}(0)\right| \leqq \pi$. Then (8.4) holds for $\theta=0$. If we keep $\epsilon<\epsilon_{0}=\left(\pi^{2} / 4\right)\left(\left(a_{2} c / b\right)(3+M)\right)^{-1}$, then the right-hand side of (8.4) is $\leqq \pi / 2$ and (8.4) holds, by continuity, for these branches for $0 \leqq t \leqq 1$. Thus we have on $E_{1}$

$$
|u| \leqq \frac{\pi}{\delta a_{1}} \frac{2 a_{2} C}{\pi b}(3+M) \epsilon=\frac{B_{1}}{\delta} \epsilon,
$$

and, if $j \geqq 0$,

$$
|j| \leqq\left(B_{1} / \delta\right) \epsilon,
$$

The same inequality holds also when $j<0$.

Thus we obtain from (8.3) and (8.5)

$$
\begin{aligned}
\left|I_{2}\right| & \leqq \frac{2^{1 / 2}}{a_{1}} \operatorname{Max}_{0 \leqq t \leqq 1}\left|\theta^{\prime}(t)-\theta_{1}^{\prime}(t)\right| \int_{t_{0}-\delta}^{t_{0}+\delta}\left|\kappa_{1}(t)\right| d t+\frac{B_{1}}{\delta} \epsilon \int_{0}^{1}\left|\kappa_{1}(t)\right| d t \\
& \leqq \frac{2\left(2^{1 / 2}\right)}{a_{1}} \frac{k \delta}{b} \operatorname{Max}_{0 \leqq t \leqq 1}\left|\theta^{\prime}(t)-\theta_{1}^{\prime}(t)\right|+\frac{k}{b} \frac{B_{1}}{\delta} \epsilon .
\end{aligned}
$$

Combining the estimates of $I_{1}$ and $I_{2}$ we find, noting that

$$
\left|\log \frac{\phi^{\prime}(0)}{\phi_{1}^{\prime}(0)}\right| \leqq \frac{M}{d} \epsilon,
$$

$$
\begin{aligned}
\operatorname{Max}_{0 \leqq t \leqq 1}|\log | \frac{\phi^{\prime}(w(t))}{\phi_{1}^{\prime}\left(w_{1}(t)\right)}|| \\
\quad \leqq\left(B_{0}+\frac{k B_{1}}{b \delta}+\frac{M}{d}\right) \epsilon+\frac{2\left(2^{1 / 2}\right)}{a_{1}} \frac{k}{b} \delta \operatorname{Max}_{0 \leqq t \leqq 1}\left|\theta^{\prime}(t)-\theta_{1}^{\prime}(t)\right| .
\end{aligned}
$$

(d) Completion of the proof. By (7.9) and (8.1), (7.3), (7.6)

$$
\begin{aligned}
\left|\theta^{\prime}(t)-\theta_{1}^{\prime}(t)\right| \leqq & || \phi^{\prime}(w(t))|-| \phi_{1}^{\prime}\left(w_{1}(t)\right)||\left|w^{\prime}(t)\right| \\
& +\left|\phi_{1}^{\prime}\left(w_{1}(t)\right)\right|\left|w^{\prime}(t)-w_{1}^{\prime}(t)\right| \\
\leqq & || \phi^{\prime}(w(t))|-| \phi_{1}^{\prime}\left(w_{1}(t)\right)|| 2 k+\left(2 a_{2} / b\right) \epsilon .
\end{aligned}
$$


Let

$$
\sigma=\operatorname{Max}_{0 \leqq t \leqq 1}|| \phi^{\prime}(w(t))|-| \phi_{1}^{\prime}\left(w_{1}(t)\right)|| .
$$

Then we obtain from (8.6) by use of the inequality

$$
|a-b| \leqq \operatorname{Max}(a, b)|\log a-\log b| \quad \text { (for } a, b>0)
$$

and of (8.1) and (7.3)

$$
\sigma \leqq \frac{a_{2}}{b}\left[\left(B_{0}+\frac{k}{b} \frac{B_{1}}{\delta}+\frac{M}{d}\right) \epsilon+\frac{2\left(2^{1 / 2}\right)}{a_{1}} \frac{k}{b} \delta\left(2 k \sigma+\frac{2 a_{2}}{b} \epsilon\right)\right] .
$$

Now we specify the value of $\delta$ so that $\left(4\left(2^{1 / 2}\right) k^{2} a_{2} / a_{1} b^{2}\right) \delta \leqq 1 / 2$; thus let

$$
\delta=\operatorname{Min}\left(1, \frac{\pi}{2 a_{2}}, \frac{a_{1} b^{2}}{8\left(2^{1 / 2}\right) k^{2} a_{2}}\right) .
$$

Thus solving (8.7) for $\sigma$, we find

$$
\sigma \leqq B_{3} \epsilon .
$$

where $B_{3}$ depends only on $d, D, c, b, k$, and $p$.

To complete the proof we need yet an estimate for $\left|e^{i \gamma}-e^{i \gamma_{1}}\right|$ where $\gamma=\arg \phi^{\prime}(w(t)), \gamma_{1}=\arg \phi_{1}^{\prime}\left(w_{1}(t)\right)$. Since $\gamma-\gamma_{1}=\tau_{1}(t)-\tau(t)+\theta(t)-\theta_{1}(t)$ $(\bmod 2 \pi)$ we have

$$
\begin{aligned}
& \left|e^{i \gamma}-e^{i \gamma 1}\right| \leqq\left|e^{i \tau}-e^{i \tau 1}\right|+\left|e^{i \theta}-e^{i \theta 1}\right| \leqq\left|\frac{w^{\prime}}{\left|w^{\prime}\right|}-\frac{w_{1}^{\prime}}{\left|w_{1}^{\prime}\right|}\right|+\left|\theta-\theta_{1}\right| \\
& \quad \leqq\left|w^{\prime}-w_{1}^{\prime}\right|\left(\frac{1}{\left|w^{\prime}\right|}+\frac{1}{\left|w_{1}^{\prime}\right|}\right)+\left|\theta-\theta_{1}\right| \leqq \frac{4}{b} \epsilon+\frac{2 c a_{2}}{\pi b}(3+M) \epsilon
\end{aligned}
$$

by (7.3), (7.6), and (8.4). Thus (7.5) follows by a combination of (8.8) with this last inequality.

We have proved the theorem under the assumption that $\epsilon \leqq \epsilon_{0}$. If $\epsilon>\epsilon_{0}$, we may take $N=2 a_{2} / b \epsilon_{0}$.

9. Corollaries of Theorem V. We first apply this theorem to derive an estimate for $\mu^{\prime}$, which, due to a stronger hypothesis, is better as to the order of $\epsilon$ than (6.3).

Corollary 1. Suppose $C$ and $C_{1}$ satisfy the hypotheses of Theorem $\mathrm{V}$ and that, in addition, for $0 \leqq t \leqq 1$,

$$
\int_{0}^{\delta}\left|w^{\prime \prime}(t+x)-w^{\prime \prime}(t)\right| \frac{d x}{x} \leqq \nu(\delta), \quad 0<\delta \leqq 1, \lim _{\delta \rightarrow 0} \nu(\delta)=0 .
$$

Then there exists a constant $A$ which depends only on $d, D, c, b, k, p$ and the "modulus of convergence" $\nu(\delta)$ such that 


$$
\mu^{\prime}=\operatorname{Sup}_{|z| \leqq 1}\left|f^{\prime}(z)-f_{1}^{\prime}(z)\right| \leqq A \epsilon .
$$

Proof. There exists a constant $m$ which depends only on $d, D, c, b, k$ and the function $\nu(\delta)$ such that for $w^{\prime}, w^{\prime \prime}$ on $C$ (see $[10$, p. 326])

$$
\left|\phi^{\prime}\left(w^{\prime}\right)-\phi^{\prime}\left(w^{\prime \prime}\right)\right| \leqq m\left|w^{\prime}-w^{\prime \prime}\right| \text {. }
$$

Now, if $z=\phi(w(t))=\phi_{1}\left(w_{1}\left(t_{1}\right)\right)$, we have, by (8.1) and (7.3),

$$
\begin{aligned}
& \left|f^{\prime}(z)-f_{1}^{\prime}(z)\right|=\left|\frac{1}{\phi^{\prime}(w(t))}-\frac{1}{\phi_{1}^{\prime}\left(w_{1}\left(t_{1}\right)\right)}\right| \leqq \frac{b^{2}}{a_{1}^{2}}\left|\phi_{1}^{\prime}\left(w_{1}\left(t_{1}\right)\right)-\phi^{\prime}(w(t))\right|, \\
& \leqq\left(\frac{b}{a_{1}}\right)^{2}\left\{\left|\phi_{1}^{\prime}\left(w_{1}\left(t_{1}\right)\right)-\phi^{\prime}\left(w\left(t_{1}\right)\right)\right|+\left|\phi^{\prime}\left(w\left(t_{1}\right)\right)-\phi^{\prime}(w(t))\right|\right\} .
\end{aligned}
$$

Thus, by (7.5) and (9.3),

$$
\begin{aligned}
\left|f^{\prime}(z)-f_{1}^{\prime}(z)\right| & \leqq\left(\frac{b}{a_{1}}\right)^{2}\left\{N \epsilon+m\left|w\left(t_{1}\right)-w(t)\right|\right\} \\
& \leqq\left(\frac{b}{a_{1}}\right)^{2}\left[N \epsilon+m\left(\left|w(t)-w_{1}\left(t_{1}\right)\right|+\left|w_{1}\left(t_{1}\right)-w\left(t_{1}\right)\right|\right)\right] \\
& \leqq\left(\frac{b}{a_{1}}\right)^{2}\left[N \epsilon+m\left|f(z)-f_{1}(z)\right|+3 \epsilon\right],
\end{aligned}
$$

by (7.7). By Theorem IV, $\left|f(z)-f_{1}(z)\right| \leqq M \epsilon$ and so we may take $A=N$ $+m M+3$.

A simple consequence of (9.2) is the following.

Corollary 2. Suppose $G$ denotes the region common to the interiors of $C$ and $C_{1}$ which contains the origin. Under the hypotheses of Corollary 1,

$$
\operatorname{Sup}_{w \in G}\left|\phi^{\prime}(w)-\phi_{1}^{\prime}(w)\right| \leqq A^{\prime} \epsilon
$$

where $A^{\prime}$ depends only on the same parameters as $A$.

The proof is obtained by an argument similar to that used above.

10. Hadamard's variation formula for the Green's function. To illustrate some of the uses of our results we outline a short proof of this formula which permits one to estimate the error obtained by neglect of the higher order terms $\left({ }^{9}\right)$. We shall apply Theorem $\mathrm{V}$ and prove it for contours with bounded curvature. This smoothness assumption may be further relaxed-at the ex-

${ }^{(9)}$ In this connection see also a derivation of Julia's variational formula for the logarithm of the mapping function recently given by R. Garnier [2], which utilizes a theorem of W. Seidel [6]. Julia's formula may be derived from that of Hadamard. Garnier does not give an explicit appraisal of the error term; such an estimate can however be obtained in a manner similar to the one described here. 
pense of the order of magnitude of the error estimate-if some of the other of our results are used instead.

Suppose $C$ and $C_{1}$ are given by the equations (7.1) and that $w_{1}(t)=w(t)$ $+i \epsilon e^{i \tau(t)} v(t)$, when $\tau(t)$ is the tangent angle to $C$ at $w(t)$ and $v(t)$ is a function such that $0 \leqq v(t) \leqq 1, v^{\prime}(t)$ has bounded difference quotients, and $\mathfrak{M}_{p}\left\{\left(e^{i \tau} v\right)^{\prime \prime}\right\}$ $\leqq 1$ for some $p>1\left({ }^{10}\right)$. Suppose furthermore, that $w(t)$ satisfies (7.2) and (7.3). For sufficiently small $\epsilon, C_{1}$ is a closed Jordan curve obtained from $C$ by displacement along the interior normals and $C_{1}$ is not exterior to $C$. Let $w_{0}$ be a point in the interior of $C_{1}$. If $\epsilon$ is sufficiently small, it is easily verified that $C$ and $C_{1}$ satisfy the hypothesis of Theorem $\mathrm{V}$ with $w_{0}$ taken as the origin.

Let, for $\zeta$ in the interior of $C_{1}, G(w, \zeta)$ and $G_{1}(w, \zeta)$ be the Green's functions of the interiors of $C$ and $C_{1}$, respectively. Then

$$
\begin{aligned}
G\left(w_{0}, \zeta\right)-G_{1}\left(w_{0}, \zeta\right) & =-\int_{C_{1}} \frac{G(w, \zeta) \partial G_{1}\left(w, w_{0}\right)}{2 \pi}\left|d w_{\mid}\right| \\
& =-\frac{1}{2 \pi} \int_{0}^{1} G\left(w_{1}(t), \zeta\right) \frac{\partial G_{1}\left(w_{1}(t), w_{0}\right)}{\partial n}\left|w_{1}^{\prime}(t)\right| d t .
\end{aligned}
$$

Since $G\left(w, w_{0}\right)=\log |\phi(w)|$ we have by Theorem V

$$
\left|\frac{\partial G\left(w(t), w_{0}\right)}{\partial n}-\frac{\partial G_{1}\left(w_{1}(t), w_{0}\right)}{\partial n}\right|=|| \phi^{\prime}(w(t))|-| \phi_{1}^{\prime}\left(w_{1}(t)|| \leqq N \epsilon .\right.
$$

Furthermore, since $\left.G^{\prime} \omega(t), \zeta\right)=0$,

$$
G\left(w_{1}(t), \zeta\right)=G\left(w_{1}(t), \zeta\right)-G(w(t), \zeta)=\left[\frac{\partial G(w(t), \zeta)}{\partial n}+o(1)\right] \epsilon v(t)
$$

by $\left[6\right.$, p. 226] or $\left[10\right.$, Theorem III*]. From the condition that $\left|w^{\prime \prime}(t)\right| \leqq k$ it follows more precisely ${ }^{(11)}$ that $|o(1)| \leqq K \epsilon \log (1 / \epsilon)$. Finally, $\left|w_{1}^{\prime}(t)\right|$ $=\left|w^{\prime}(t)\right|+O(\epsilon)$. Hence we obtain Hadamard's formula

$$
\begin{aligned}
G\left(w_{0}, \zeta\right)-G_{1}\left(w_{0}, \zeta\right) & =-\frac{1}{2 \pi} \int_{0}^{1} \frac{\partial G(w(t), \zeta)}{\partial n} \frac{\partial G\left(w(t), w_{0}\right)}{\partial n} \epsilon v(t)\left|w^{\prime}(t)\right| d t+o(\epsilon) \\
& =-\frac{1}{2 \pi} \int_{C} \frac{\partial G(w, \zeta)}{\partial n} \frac{\partial G\left(w, w_{0}\right)}{\partial n} \delta n|d w|+o(\epsilon)
\end{aligned}
$$

10) The normalizations $v \leqq 1$ and $\mathfrak{M}_{p}\left\{\left(e^{i \tau} v\right)^{\prime \prime}\right\} \leqq 1$ are, of course, unessential.

(11) Consider $F(z)=\log \left|f^{\prime}(z)\right|+i \arg f^{\prime}(z)$ where $f(z)$ maps $|z|<1$ onto the interior of $C$ such that $f(0)=\zeta, f^{\prime}(0)>0$ and $\arg f^{\prime}(0)=0$. Let $\psi(w)$ denote the inverse of $f(z)$. Because of the hypothesis $\left|w^{\prime \prime}(t)\right| \leqq k, \operatorname{Im}[F(z)]$ satisfies a Lipschitz condition, and it follows then from a well known theorem of Privaloff $\left[16\right.$, p. 157] that, for $\left|z_{1}\right|=\left|z_{2}\right|=1,\left|F\left(z_{2}\right)-F\left(z_{1}\right)\right| \leqq M\left|z_{2}-z_{1}\right|$ $\cdot|\log | z_{2}-z_{1}||$, where $M$ is a constant. The use of the Phragmén-Lindelöf theorem shows then the validity of this inequality when $\left|z_{1}\right|=1$ and $\left|z_{2}\right| \leqq 1$. From this result one infers in turn that $\left|\psi^{\prime}(w)-\psi^{\prime}\left(w_{0}\right)\right| \leqq M_{1}\left|w-w_{0}\right||\log | w-w_{0}||$, for $w_{0} \in C, w \in I(C)$. This, together with the fact that $\left|\psi\left(w_{0}\right)-\psi(w)\right| \leqq M_{2}\left|w_{0}-w\right|$, proves our statement. If $C$ is contained in the ring $d \leqq|w-\zeta| \leqq D$, then an examination of the steps of this proof shows that the constants $M, M_{1}$, $M_{2}$ depend only on $d, D, c, k$ and the lower bound $b$ for $\left|w^{\prime}(t)\right|$ in (7.3). 
where $\delta n=\epsilon v(t)$ and $|o(\epsilon)| \leqq K_{1} \epsilon^{2} \log (1 / \epsilon)$. If the condition (9.1) is added to the hypotheses on $w(t)$, then $|o(\epsilon)| \leqq K_{2} \epsilon^{2}$ as may be seen by use of $[10$, Theorem III] applied for $n=2$.

The fact that the constants $N$ in (10.1) and $M, M_{1}, M_{2}$ in( (11) depend only on the parameters named above shows that $K$ and $K_{1}$ may be determined uniformly for all $\zeta$ and $w_{0}$ in any fixed closed domain contained in the interior of $C$. The same holds also for $K_{2}$.

It is not essential that $C_{1}$ be in the interior of $C$ or that the "displacement" be taken in the direction of the normal. A slight modification of the above argument provides a proof of the Hadamard formula for the general case.

\section{REFERENCES}

1. L. Fejér and F. Riesz, Über einige funktionentheoretische Ungleichungen, Math. Zeit. vol. 11 (1921) pp. 305-314.

2. René Garnier, Sur la variation de la représentation conforme d'un domaine variable, Rendiconti di Matematica e delle sue applicazioni (V) vol. 14 (1954) pp. 258-267.

3. G. H. Hardy and J. E. Littlewood, A maximal theorem with function-theoretic applications, Acta Math. vol. 54 (1930) pp. 81-116.

4. Fulton Koehler, A note on neighboring Jordan curves, to appear in American Mathematical Monthly.

5. M. Riesz, Sur les fonctions conjuguées, Math. Zeit. vol. 27 (1927) pp. 218-244.

6. W. Seidel, Über die Ränderzuordnung bei konformen Abbildungen, Math. Ann. vol. 104 (1931) pp. 182-243.

7. B. von Kerekjarto, Vorlesungen über Topologie, Berlin, Springer, 1923.

8. S. E. Warschawski, Über einige Konvergenzsätze aus der Theorie der konformen Abbildung, Nachrichten von der Gesellschaft der Wissenschaften, Göttingen, Math. Phys. Klasse, 1930, pp. 344-369.

9. - Über das Randverhalten der Ableitung der Abbildungsfunktion bei konformer Abbildung, Math. Zeit. vol. 35 (1932) pp. 321-456.

10. - On the higher derivatives at the boundary in conformal mapping, Trans. Amer. Math. Soc. vol. 38 (1935) pp. 310-340.

11. - On the degree of variation in conformal mapping of variable regions, Trans. Amer. Math. Soc. vol. 69 (1950) pp. 335-356.

12. - On conformal mapping of regions bounded by smooth curves, Proc. Amer. Math. Soc. vol. 2 (1951) pp. 254-261.

13. - On conformal mapping of variable regions, National Bureau of Standards Applied Mathematics Series, vol. 18, 1952, pp. 175-187.

14. - On mean convergence in conformal mapping, Archiv der Mathematik vol. 6 (1955) pp. 102-114.

15. A. Zygmund, Sur les fonctions conjuguées, Fund. Math. vol. 13 (1929) pp. 284-303.

16. —-, Trigonometrical series, Warsaw, 1935.

UNIVERSITY OF MiNNESOTA, MinNEAPOLIS, MinN. 